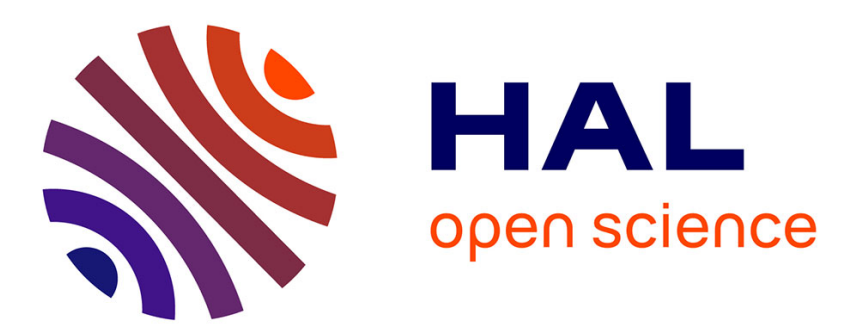

\title{
Electro-optic delay oscillator with nonlocal nonlinearity: Optical phase dynamics, chaos, and synchronization
}

\author{
R. Lavrov, M. Peil, M. Jacquot, L. Larger, V.S. Udalstov, J.M. Dudley
}

\section{To cite this version:}

R. Lavrov, M. Peil, M. Jacquot, L. Larger, V.S. Udalstov, et al.. Electro-optic delay oscillator with nonlocal nonlinearity: Optical phase dynamics, chaos, and synchronization. Physical Review E: Statistical, Nonlinear, and Soft Matter Physics, 2009, 80 (2), pp.026207. 10.1103/PhysRevE.80.026207 . hal-00453016

\section{HAL Id: hal-00453016 https://hal.science/hal-00453016}

Submitted on 12 Apr 2021

HAL is a multi-disciplinary open access archive for the deposit and dissemination of scientific research documents, whether they are published or not. The documents may come from teaching and research institutions in France or abroad, or from public or private research centers.
L'archive ouverte pluridisciplinaire HAL, est destinée au dépôt et à la diffusion de documents scientifiques de niveau recherche, publiés ou non, émanant des établissements d'enseignement et de recherche français ou étrangers, des laboratoires publics ou privés. 


\title{
Electro-optic delay oscillator with nonlocal nonlinearity: Optical phase dynamics, chaos, and synchronization
}

\author{
Roman Lavrov, Michael Peil, Maxime Jacquot, Laurent Larger, Vladimir Udaltsov, and John Dudley \\ Department of Optics, UMR CNRS FEMTO-ST 6174, University of Franche-Comté, 16 Route de Gray, 25030 Besançon Cedex, France
}

(Received 7 September 2008; published 19 August 2009)

\begin{abstract}
We demonstrate experimentally how nonlinear optical phase dynamics can be generated with an electrooptic delay oscillator. The presented architecture consists of a linear phase modulator, followed by a delay line, and a differential phase-shift keying demodulator (DPSK-d). The latter represents the nonlinear element of the oscillator effecting a nonlinear transformation. This nonlinearity is considered as nonlocal in time since it is ruled by an intrinsic differential delay, which is significantly greater than the typical phase variations. To study the effect of this specific nonlinearity, we characterize the dynamics in terms of the dependence of the relevant feedback gain parameter. Our results reveal the occurrence of regular GHz oscillations (approximately half of the DPSK-d free spectral range), as well as a pronounced broadband phase-chaotic dynamics. Beyond this, the observed dynamical phenomena offer potential for applications in the field of microwave photonics and, in particular, for the realization of novel chaos communication systems. High quality and broadband phase-chaos synchronization is also reported with an emitter-receiver pair of the setup.
\end{abstract}

DOI: 10.1103/PhysRevE.80.026207

\section{INTRODUCTION}

Optical chaos communications using chaotically emitting semiconductor lasers were first proposed 15 years ago [1-3]. This approach makes use of the intrinsic nonlinear interaction of the light field and the gain material of the semiconductor laser. This interaction does not yield, in itself, chaotic intensity output of the laser. However, the combination of this nonlinear interaction and a delayed feedback [4-6], which can either be optical or electrical, can induce complex chaotic dynamics, since it renders the system mathematically infinite dimensional. Therefore, in recent years, much research has been dedicated to investigate the dynamical properties of semiconductor lasers which are subject to delayed feedback or to external perturbation in form of optical injection or electrical driving. Meanwhile, numerous dynamical phenomena and regimes have been theoretically and experimentally identified and analyzed, including regimes of complex chaotic dynamics. For an overview, we refer to [7].

Furthermore, with these laser systems, also chaos synchronization has been extensively investigated and demonstrated. It has been shown that a receiver system which is synchronized to a chaotic transmitter system can discriminate between the chaotic signal and a message that has been applied in form of an external perturbation to the transmitter system [8-11]. Utilizing this mechanism, feasibility of optical chaos communication systems (encrypted communications) has been demonstrated. The required chaos synchronization is, however, very sensitive to the physical parameters of the lasers which on one hand is desired for reasons of security, but on the other hand it is still challenging to achieve the required parameter agreement of the lasers at the fabrication stage. Very recent work led to successful demonstration of integrated photonic devices explicitly designed for chaos generation and synchronization [12]. With this photonic integrated device approach, accurate parameter control is expected at the fabrication stage, thus taking an important step toward the definition of a physical hardware key for secure chaos communications.
PACS number(s): 05.45.Gg, 05.45.Jn, 05.45.Xt, 42.65.Sf

Out of this laser dynamics approach for practical optical chaos generation, another approach based on electro-optic and optoelectronic nonlinear delayed feedback oscillators has been proposed [13-15]. This approach was following the Ikeda's idea [16] on optical chaos generation principles. The differential process itself is linear in this Ikeda-like dynamics, since it originates from linear dissipative filtering. The filtering effect is often related to unavoidable but controllable electronic bandwidth limitations in the case of optoelectronic or electro-optic realizations. In all-optical systems as for the original Ikeda ring cavity setup, the linear filtering originates from the dissipative filtering effect of the level lifetime in the light-matter interaction process [16]. Ikeda-like chaotic behavior is still induced by application of a delayed nonlinear feedback term, but in that case, this nonlinear term is driving the linear differential process. A typical nonlinear transformation is the one obtained when modulating the interference condition of a two-wave interferometer. This is realized in the Ikeda ring cavity as the input light beam interferes with the cavity feedback light beam, which phase is Kerr modulated by the previous round intensity interference. With the electro-optic version of the Ikeda dynamics, the interference nonlinear modulation transfer function is performed between the electrical input and the optical output of an integrated optics electro-optic Mach-Zehnder modulator. The required delay is practically implemented by a given length of a light propagation medium: the cavity length for the original Ikeda setup or a fixed length of fiber for its optoelectronic version. The whole oscillator is formed by the interconnected individual elements, realizing a delayed feedback system capable of chaotic behavior. When compared to laser-dynamicsbased systems, the electro-optic and optoelectronic approaches showed comparable or currently even slightly better $[17,18]$ performances in terms of bit rate capability (up to $3.2 \mathrm{~Gb} / \mathrm{s}$ ) and in terms of decoding quality [measured by the bit error rates (BERs), better than $10^{-9}$ ]. From the experimental point of view, the so far realized electro-optic chaos generators have proven their value regarding two important issues: the practical investigation of fundamental dynamical 
properties exhibited by nonlinear delay systems and also as a first successful demonstration of efficient chaos-based optical communication systems. All those electro-optic experiments have been however performed with interconnected discrete devices. Their integration potential is not yet exploited and is under current investigation. Highly integrated systems could be realized in the future, e.g., using semiconductor-based electro-optic modulators.

The results reported in the present paper lie within the latter research area on the electro-optic approach for chaos communications. A system architecture is proposed, which exploits recent ideas and potentials in optical phase modulation (instead of intensity modulation) for fiber optics communications.

The paper is organized as follows. First, we introduce the general principle of the dynamical process which we apply. The reported architecture is discussed and compared to earlier realizations of electro-optic and optoelectronic chaos generators. Then, in Sec. II, we describe in detail the experimental setup which realizes the desired dynamical process for the optical phase of a light beam. The dynamical properties of the experiment will be characterized in Sec. III in terms of the major relevant parameter: the feedback gain of the oscillator. We will study and discuss the emergence of different dynamical regimes through the analysis of the corresponding temporal wave forms, amplitude probability density functions (PDFs), and rf spectra. Before concluding, in Sec. IV, we will propose an encoding and decoding scheme that can be applied for the realization of an optical chaos communication link on the basis of the reported phase chaos generator. Specifically, we demonstrate an experimental optical phase chaos synchronization $[19,20]$, in which chaos cancellation is achieved directly in the optical domain. For this, we show that excellent synchronization quality can be achieved experimentally, by demonstrating very good cancellation of the received chaotic signal from the transmitter, when the synchronized chaotic receiver signal is subtracted.

\section{EXPERIMENTAL SETUP}

\section{A. Principle of operation}

Ikeda-based nonlinear delay dynamics can be usually reduced in their modeling to a scalar nonlinear delay differential equation of the form

$$
x(t)+\tau \frac{d x}{d t}(t)=f_{\mathrm{NL}}[x(t-T)],
$$

where the left-hand side is representative of a linear filtering process with a characteristic time $\tau$. The right-hand side is the nonlinear delayed feedback term involving the nonlinear transformation $f_{\mathrm{NL}}$. The time delay $T$ is usually much greater than the characteristic time $\tau$. When tuned into chaotic regime, the dimension of the attractor is known to increase proportionally to $T / \tau$ [21-23], which can be easily as high as several hundreds in practical situations. In previous attempts to perform the Ikeda ring cavity in optics (or optoelectronics, electro-optics), this nonlinear transformation was implemented as an electronically tunable two-wave interference function; the optical intensity at the output of the nonlinear transformation was thus ruled as a $\cos ^{2}$ transformation of the interferometer phase difference $\varphi=2 \pi \Delta / \lambda$ ( $\Delta$ is the optical path difference and $\lambda$ the optical wavelength). The latter phase $\varphi$ was modulated over at least $\pi$ to scan a strongenough nonlinear range of the $\cos ^{2}$-transfer function. This was obtained through the modulation of an electronic quantity driving linearly either $\Delta$ (through the electro-optic effect $[13,14]$ ) or $\lambda$ (through the linear wavelength modulation capability of a multielectrode tunable distributed Bragg reflector (DBR) laser [15]). The dynamical properties of these setups are captured by the same mathematical modeling as in Eq. (1), which indeed also exhibit very rich dynamics, similar than the ones first predicted by Ikeda. It is also common for these systems that the corresponding nonlinearity is described by a $\cos ^{2}$-shaped function, which is defined locally in time, i.e., which is memoryless $\left(f_{\mathrm{NL}}\right.$ is an instantaneous function of $x$ only and not of time).

In our attempt to develop fast chaos communication systems, our group concentrated its efforts on the Neyer and Voges setup [14], which was making use of potentially very fast integrated optics devices, e.g., $\mathrm{LiNbO}_{3}$ electro-optic Mach-Zehnder modulators. Our investigations led to the demonstration of the attractive potential of this approach for chaos communications [17]. The success of the demonstration was, however, obtained at the cost of relatively complex polarization state control in a polarization combiner. This combiner was performing an all-optical mixing process between the two crossed polarization light beams of the chaotic optical intensity carrier and the optical intensity binary data, respectively. For security issues, fast polarization scrambling was also required to prevent from a simple polarization splitting attack on the transmission line. The practical issue causing this additional experimental complexity is the one concerned by the mixing technique between the chaotic dynamics with the message. When working with the physical variable "intensity," this issue becomes critical because adding optically two intensity fluctuations of the same wavelength generally leads to undesired interference phenomena: the mixing process is not linear anymore and the decoding process involving electronic subtraction of photodiode output signals (the received and the synchronized one) cannot be applied. The use of crossed polarization could thus prevent from the interference phenomena, but at the cost of additional polarization scrambling due to security issues.

In order to circumvent this practical drawback of chaotic optical intensity, we studied the possibility for the realization of an optical phase chaos generator. A linear superposition between a chaotic phase modulation and a standard binary differential phase modulation can be much more easily and efficiently implemented, e.g., with two cascaded phase modulators. The design of a phase chaos oscillator was then investigated on the basis of our accumulated experience on intensity chaos generation through the Ikeda principles. The next section will detail the practical architecture developed from these investigations. It will underline the very specific dynamical features that are associated with this new electrooptic chaos generation principle. It involves fast phase modulation and imbalanced passive interferometers, which time imbalance is longer than the characteristic time of the 


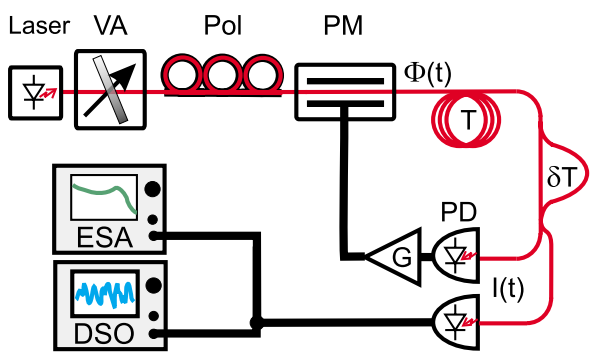

FIG. 1. (Color online) Experimental setup of the electro-optic nonlinear delay phase oscillator.

phase modulation. That specific situation is referred as the temporal nonlocality of the nonlinear phase-to-intensity conversion or, differently speaking, the imbalanced dynamical interference phenomena. One could say equivalently that the interference condition can be dynamically modulated by the phase modulation as soon as the latter is faster than the interferometer time imbalance.

\section{B. Differential phase-shift keying delay oscillator}

A scheme of the experimental setup for the electro-optic nonlinear delay phase oscillator is shown in Fig. 1. In this setup, a $1.55 \mu \mathrm{m}$ distributed feedback (DFB) semiconductor laser with a narrow linewidth of $\Delta \lambda<2 \mathrm{MHz}$ is used for injection of a continuous-wave (cw) light with power 0 $<P_{0}<3.2 \mathrm{~mW}$ into the delayed feedback loop of the electro-optic oscillator. A variable attenuator (VA) is used for adjusting the injected intensity without changing the oscillation frequency of the source (which would occur if the laser current is used). Laser frequency modifications due to injection current variations are indeed critical for the operating conditions of the phase-to-intensity (imbalanced interferometer) demodulator.

The cw-injected light enters a broadband phase modulator (PM) with a bandwidth of $20 \mathrm{GHz}$. The PM represents a key element of the feedback loop as a fast electrical-to-optical converter. The optical phase of the injected $\mathrm{CW}$ light is thus modulated according to the voltage applied to the electrical rf input of the PM, while the intensity of the output light remains constant.

This output light is delayed by propagation in a few meters of optical fiber until it enters a fiber-based passive imbalanced interferometer. For this, we used a commercial differential phase-shift keying demodulator (DPSK-d) with a $2.5 \mathrm{GHz}$ free spectral range (FSR, corresponding to a time imbalancing of $\delta T \approx 400 \mathrm{ps}$ ) performed by a fiber-based Mach-Zehnder interferometer (MZI) in an athermal packaging. It should be noticed here that in principle, any customized imbalanced multiple wave interferometer could be used as long as the characteristic free spectral ranges attached to the imbalancings are smaller than the PM modulation bandwidth and if the interference contrast is reasonably good $(>6 \mathrm{~dB})$. The interferometer is aimed at performing a nonlinear, dynamical (nonlocal in time), phase-to-intensity conversion.

The phase-to-intensity conversion is governed by a nonlinear transformation described by the function $P_{\text {out }}(t)$ $\left.\sim \beta \cos ^{2}\left[\Phi_{\text {in }}(t)-\Phi_{\text {in }}(t-\delta T)+\Phi_{0}\right)\right]$. In this notation, $P_{\text {out }}(t)$ stands for the optical power at the MZI output, $\Phi_{\text {in }}$ for the optical phase at the input, and $\Phi_{0}$ for the offset interference phase of the MZI (depending on the optical path difference in the MZI, as well as on the laser frequency). An important difference in this nonlinear transformation is its nonlocal character in time compared to the standard Ikeda-like dynamics. For the reported phase chaos dynamics, a differential term is involved as the argument of the $\cos ^{2}$ function, thus inducing an implicit dynamical character in the nonlinear transformation, $f_{\mathrm{NL}}(x, t, t-\delta T)$. The constant $\beta$ depends on the optical input power, on the optoelectronic and electrooptic conversion efficiencies, and on the electronic amplification factor.

The obtained MZI output intensity signal is detected with a broadband amplified photodetector (PD) with a bandwidth of $30 \mathrm{kHz}-13 \mathrm{GHz}(-3 \mathrm{~dB}$ cutoff) and a conversion efficiency of $S=1.9 \mathrm{~V} / \mathrm{mW}$. It is the electrical component with the smallest bandwidth in the oscillator loop, therefore it effectively determines the overall bandwidth of the combined bandpass-filtering properties of the system. Accordingly, the characteristic time scales of the bandpass filter are $\tau=12.2 \mathrm{ps}$ for the high cutoff (which indeed satisfies $\tau$ $\ll \delta T)$ and $\theta=5.3 \mu$ s for the low cutoff; the corresponding frequencies are $\nu_{\mathrm{hf}}=(2 \pi \tau)^{-1}$ and $\nu_{\mathrm{lf}}=(2 \pi \theta)^{-1}$, respectively. The converted electrical signal is then linearly amplified with a broadband amplifier which drives the rf input of the PM. It has a gain of $18 \mathrm{~dB}$ (a linear amplitude amplification of 8) and a bandwidth of $30 \mathrm{kHz}-25 \mathrm{GHz}$. Thus, the optical phase of the light at the PM output is modulated according to its own delayed history. The electro-optic effect is closing the delayed feedback loop of the nonlinear oscillator. The total delay time comprising the optical and electrical delays corresponds to $T=24.35 \mathrm{~ns}$. Since the maximum output voltage of the $\mathrm{rf}$ driver is $13.0 \mathrm{~V}$ and since the half wave voltage of the PM corresponds to $V_{\pi}=4.0$, the phase $\Phi_{\text {in }}(t)$ can be modulated by up to $3.25 \pi \mathrm{rad}$. Up to three extrema of the nonlinear $\cos ^{2}$-interference function can thus be swept in the DPSK-d. This strong nonlinear operation capability is particularly of interest when complex chaotic waveforms are desired, typically for chaos communication applications where very low autocorrelation carriers are desired.

According to the previous description of the electro-optic delay nonlinear phase oscillator, a simple dynamical model can be established. The band-limited electronic feedback bandwidth is responsible for an integrodifferential linear dynamical process, which is driven by the nonlinear delayed term issued from the phase-to-intensity conversion of the DPSK-d. The dynamical equation ruling the phase chaos generator reads, according to this simple modeling (see also Refs. [17,24] where similar bandpass dynamical models are used),

$$
\begin{aligned}
& \frac{1}{\theta} \int_{t_{0}}^{t} x(\xi) d \xi+x(t)+\tau \frac{d x}{d t}(t) \\
& \quad=\beta \cos ^{2}\left[x(t-T)-x(t-T-\delta T)+\Phi_{0}\right],
\end{aligned}
$$

where $x(t)$ is proportional to the voltage driving the modulator. This normalized dynamical variable can be viewed also 
as proportional to the optical phase modulation $\Phi_{\text {in }}(t)$ induced by the electro-optic modulator, since the latter is assumed to have a quasiadiabatic response compared to other slower limiting dynamical elements (typically the amplified photodiode). For similar reasons concerning the even broader bandwidth of the rf amplifier driving the PM, the same signal can be considered as proportional to the photodiode output. This is a consequence of the fact that the photodiode performs the strongest filtering in the oscillator loop. This filtering is operated on the detected optical intensity fluctuations at the output of the DPSK-d, i.e., which is mathematically represented by the nonlinear delayed term in the right-hand side of Eq. (2).

Equation (2) will be later used to support through numerical integration: the proposed modeling of the optical phase electro-optic delay oscillator. It should be noticed that this equation differs from the usual Ikeda delay dynamics essentially in two points. First, the right-hand side involves a difference dual delay $(T$ and $\delta T)$ nonlinear term. Since two different delays are involved in the nonlinear term, this one is qualified as nonlocal in time. In our practical situation, the second time delay $\delta T$ is much shorter (a factor of 200) than the long delay $T$, but it is also larger (a factor of 40) than the high cutoff time $\tau$. The second point differing from the Ikeda dynamics is less unusual since it has been already considered in the literature, but its fundamental nonlinear dynamical issues have been only recently reported [24,25]: it is concerned by the integrodifferential character of the left-hand side, which is a consequence of the bandpass character of the linear filtering. This is opposed to the low-pass (or dcpreserving) filtering, typically considered in Ikeda-like dynamics, and issuing a differential term only. It should be noticed here that the first-order differential term and the single integration term in the left-hand side of Eq. (2) are representative of the following assumption: the bandpass filter is resulting from two cascaded first-order low-pass (with the cutoff time $\tau$ ) and high-pass (with cutoff time $\theta$ ) filters. This is definitely not as simple in a real-world broadband dynamics as the one of concern here, since especially the low-pass cutoff is of a much higher order (the actual measure of the linear filtering function by a $40 \mathrm{GHz}$ vectorial analyzer revealed an equivalent tenth-order low-pass cutoff). Such higher-order cutoff have been indeed considered for the numerical simulations presented later and performed with time domain digital filters. This higher-order frequency cutoff corresponds mathematically in Eq. (2) to additional $n$ th-order derivative terms, $n=1-10$ (and eventually also additional higher-order integral terms depending on the high-pass cutoff order). However, if analytical investigations are concerned, it has been recently found [24] that the qualitative dynamics can indeed be captured by the simple and analytically tractable first-order integral and first-order derivative model of Eq. (2). Notice that higher orders seem to be crucial for some specific dynamical features as it was underlined in [24].

For experimental characterization of the optical phase dynamics in the intensity domain, we use the complementary output of the MZI ( $\sin ^{2}$ output) at which we detect the light with another similar broadband photodetector. This photodetector is of the same kind as the one used within the feedback loop. Therefore, we detect the complementary signal with respect to the one amplified and used as the feedback voltage driving the PM. The externally detected signal is recorded and analyzed in the temporal and in the rf spectral domains. This is done simultaneously using a digital storage oscilloscope (DSO) with $12 \mathrm{GHz}$ analog bandwidth and a sampling rate of $40 \mathrm{GSamples/s}$ and with an electrical spectrum analyzer (ESA) with a bandwidth of $22 \mathrm{GHz}$, respectively.

\section{CHARACTERIZATION OF THE DYNAMICS}

For characterizing the dynamical properties of the oscillator, we finely tune the laser frequency $\nu=c / \lambda$ to set the offset interference phase of the MZI to $\Phi_{0}=\pi(\delta T) \nu=\pi / 4$. This fine tuning, as well as a precise control and stabilization of this offset phase parameter, can also be achieved by thermally tuning the differential delay $(\delta T)$ through a heating wire integrated in the commercial DPSK-d. The parameter stability of the DPSK-d operating point is practically enough over several tens of minutes if the device environment is carefully protected. If longer stability is desired, active control of the DPSK-d operating point is useful.

It can be noticed that the chosen (arbitrary) operating point $\left(\Phi_{0}=\pi / 4\right)$ corresponds to the middle situation with respect to the constructive and the destructive interference situation (in the standard use for DPSK transmission, the typical operating point is the destructive or the constructive one). The value of $\Phi_{0}=\pi / 4 \bmod (\pi)$ is favorable when low feedback gain instabilities are explored, since the steadystate dynamics operates at the maximum slope of the nonlinear function, for which first instabilities are expected even for low values of the feedback gain.

The observation of the dynamical behavior is performed via time series and their corresponding rf spectra. The bifurcations of the behavior are obtained for increasing the injection power $P_{0}$, which is equivalent to a linearly increasing feedback gain. In the following, this parameter will be substituted by a normalized feedback gain parameter which also takes into account the electrical amplification, defined by $\beta$ $=\pi \gamma \alpha G S P_{0} /\left(2 V_{\pi}\right)$. With the actual parameter values of the setup, $\beta$ can be varied from 0 to 5.1 , the highest value corresponding to the already mentioned $3.25 V_{\pi}$ of the electrooptic (EO) voltage swing.

Figure 2 depicts examples of measured temporal dynamics, both for long time scales (a few delay times $T$, left figures) and shorter ones (a few DPSK imbalance times $\delta T$, right figures). These time traces give rise to different dynamical states depending on the feedback gain. When $\beta$ is small, a stable zero steady state is observed as expected. At $\beta=0.5$, the steady states lose its stability and a limit cycle appears. The usual threshold for this Hopf bifurcation is typically 1 for the Ikeda dynamics. We clearly see here that the difference term $[x(t)-x(t-\delta T)]$ lowers by a factor of 2 the Hopf threshold, with a frequency which must be related to both $\delta T$ and $T$. The typical oscillation is represented in Figs. 2(a) and 2(d) for $\beta=0.6$; it corresponds to a rapid oscillation between a two level states, with tilted decreasing plateaus. The observed oscillation frequency is clearly related to the DPSK-d. The rf amplitude filtering of the DPSK-d, when applied to a phase modulated light beam, gives a maximum 


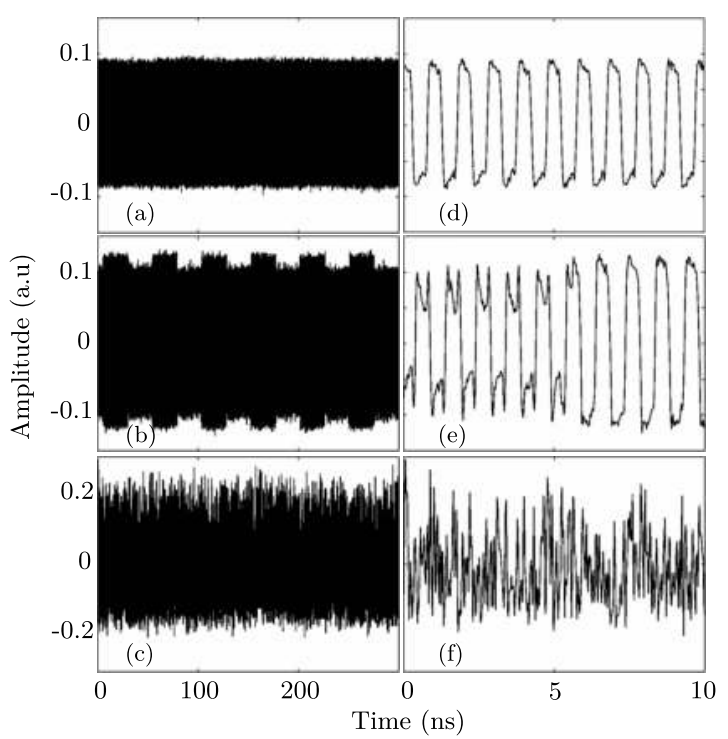

FIG. 2. Experimental time series for different values of the normalized feedback gain $\beta$ detected at the output of the MachZehnder DPSK-d for $\Phi_{0}=\pi / 4$. (a)-(c) are zoomed in (d)-(f). In (a) $/($ d) $\beta \simeq 0.6$, (b)/(e) $\beta \simeq 1.3$, and (c) $/($ f) $\beta \simeq 5.1$.

transmission around $1.25 \mathrm{GHz}$ (modulo $2.5 \mathrm{GHz}$ ), which is half of its FSR (a zero transmission occurs at zero frequency and at the FSR modulo itself). The actually observed $1 \mathrm{GHz}$ oscillation frequency is the combined $\mathrm{rf}$ filtering of the DPSK-d together with the amplitude response of the electronic feedback (amplified photodiode+rf driver+PM electrodes). We have checked that the linear open loop rf filtering exhibits a maximum at around $1 \mathrm{GHz}$, instead of FSR/2, thus explaining the actual closed-loop oscillation frequency.

When the feedback gain is slightly increased, a secondary bifurcation of a Neimark-Sacker type is observed. Such a bifurcation is already known for delay systems and it has been analyzed for other physical setups [26,27]. A typical corresponding time trace is represented in Figs. 2(b) and $2(\mathrm{e})$, which is obtained for $\beta \simeq 1.3$. The dynamics is now characterized by both the fast $1 \mathrm{GHz}$ oscillation (e) as well as a $2 T$-periodic envelope modulation (b). This kind of bifurcation was only recently observed and demonstrated analytically in [25] for the particular case of the intensity EO dynamics with a bandpass selective feedback. This setup was initially proposed in [14] in order to perform experimentally an Ikeda-like dynamics, with a low-pass delayed feedback. The bandpass selective version studied in [25] is well known in the context of the so-called optoelectronic oscillators, intended for high spectral purity microwave generation. It involves very long fiber delay lines and also highly selective feedback filtering around the desired microwave oscillation frequency. In the setup proposed in this paper, the EO MZI is replaced by a PM, followed by an imbalanced DPSK-d. An analogy can be drawn between the two setups in the sense that the DPSK-d plays the role of a "relatively" narrow-band spectral filtering, forcing the rf oscillation within its bandwidth around $1.25 \mathrm{GHz}$. Additionally, in order to support the Neimark-Sacker interpretation preferentially to the higher harmonic synchronization one [28], the following physical argument can be proposed: the two successively appearing

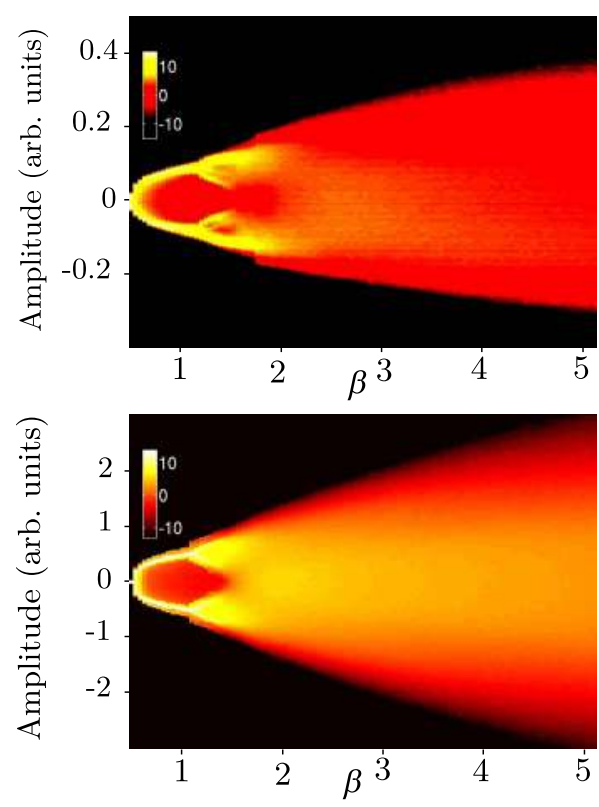

FIG. 3. (Color online) Bifurcation diagrams of the amplitude probability density functions (logarithmic color scale) of the dynamics vs normalized feedback gain $\beta$. Upper: from experimental time traces; lower: from computed time series using Eq. (2).

frequencies are clearly related to two independent and very different physical time scales-the DPSK-d imbalancing $\delta T$ [fast oscillations in Fig. 2(e)] and the total delay $T$ [slow envelope seen in Fig. 2(b)].

When higher feedback gains are concerned, the phase chaos generator however exhibits significantly different dynamical features compared to [25]. The time traces observed with the maximum feedback gain $\beta \simeq 5.1$ are represented in Figs. 2(c) and 2(f). They show large amplitude chaotic fluctuations corresponding to a broadband spectrum covering the full bandwidth of the electronic feedback (see Fig. 6).

The amplitude probability density function (PDF) is then used as a graphical representation of a given temporal dynamics. When finely scanning the bifurcation parameter $\beta$, a bifurcation diagram can be plotted to represent a quasicontinuous evolution of the dynamics through its PDF with respect to $\beta$. This is represented in Fig. 3, where the PDF is practically encoded via a logarithmic color scaling (white for highly probable amplitudes and dark for low probability amplitudes, though red for intermediate PDF amplitudes). The same kind of such bifurcation diagram is drawn for both experimental (upper) and numerical (lower) time traces. The experimental time trace duration is $4 \mu \mathrm{s}$ at 40 GSamples/s, thus leading to 160000 points used for calculating the amplitude distribution (the corresponding time trace is of course a filtered image of the actual dynamics, due to the limited bandwidth of the real time sampling oscilloscope). The numerical diagram was making use of around 400000 points over a comparable duration, computed with a time domain digital filter. As already noticed, the digital filter used for the numerical integration is fitting the actual strength of the frequency cutoff (tenth order for the high-frequency low-pass cutoff and first order for the low-frequency high-pass cutoff). The low-pass digital filter is of a Butterworth kind. 


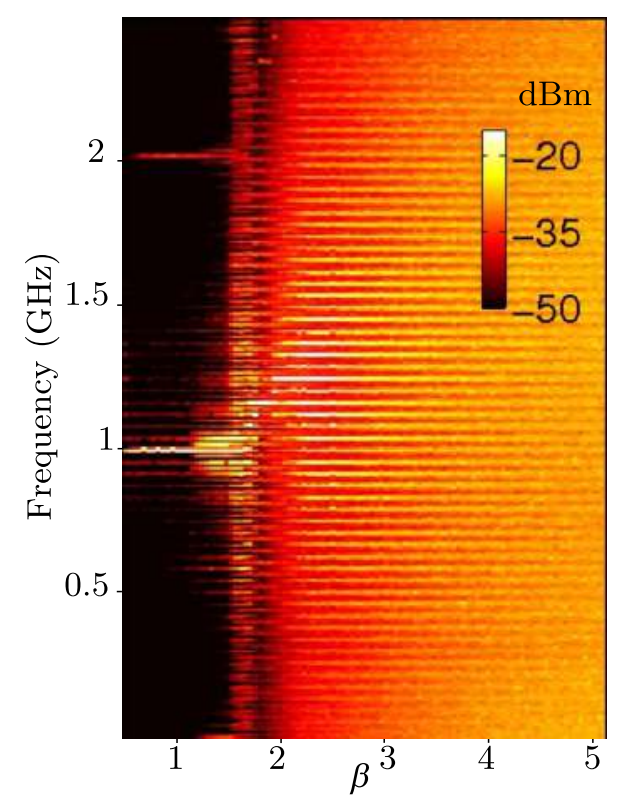

FIG. 4. (Color online) Low frequency range spectral bifurcation diagram. Color-encoded amplitudes are in $\mathrm{dB}$.

It can be noticed that both diagrams show a very good resemblance at least qualitatively but also quantitatively in terms of bifurcating values of $\beta$. Such diagrams allow giving a summarized picture of the bifurcation scenario met by the optical phase dynamics, while increasing $\beta$ from the stable steady state to the fully developed chaotic regime. This bifurcation scenario can be described as follows.

For very low feedback gains, the oscillator is nearly an open loop one and the zero amplitude steady state is stable (this part of the diagram is however not represented in Fig. 3, since it corresponds to the trivial horizontal segment at the zero vertical level, spanning from $\beta=0$ to 0.5 ). From $\beta$ $\geq 0.5$ up to 1.3 , one can identify a two-level oscillation appearing through a Hopf bifurcation, with a frequency related to FSR/2. A typical time trace of this limit cycle was given in Figs. 2(a) and 2(d). The cycle amplitude grows continuously from zero, while $\beta$ is increased (the Hopf is supercritical). Between $1.3 \leq \beta \leq 1.7$, a four-amplitude level is revealed by the bifurcation diagram, as was also described through the time traces in Figs. 2(b) and 2(e). As pointed out, this fourlevel oscillation is not issued from a period doubling as it is for standard Ikeda dynamics, but rather from the occurrence of an additional frequency through a Neimark-Sacker bifurcation (limit cycle to torus). From 1.7 to 1.9 , the experimental bifurcation diagram shows two positive and negative bands of oscillation amplitudes, separated by a low probability amplitude band at the central low-amplitude levels. This transition dynamics from periodic to chaotic regimes resemble the prechaotic states of the inverse cascade typically observed with Ikeda-like dynamics. One can notice here a difference with the numerical bifurcation diagram, which shows a very small $\beta$ range concerned by these transition dynamics. For higher gain $(>1.9)$, we find a washing out of the amplitude characteristics, so that the PDFs develop into widespread almost-Gaussian-shaped functions. This feature is also known to exist for chaotic dynamics of nonlinear
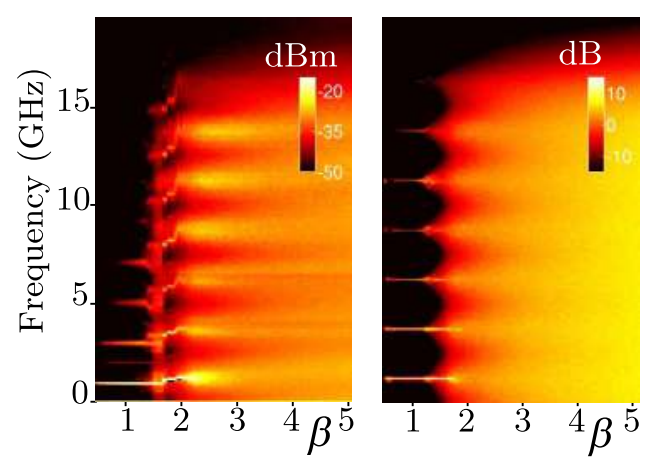

FIG. 5. (Color online) Spectral bifurcation diagram in the highfrequency range. Left: experimental; right: numerical.

delayed feedback systems modeled by standard single delay dynamics $[22,29]$.

Additional information about this bifurcation scenario and the properties of the visited dynamical regimes can be obtained from a spectral analysis. Therefore, we plot the corresponding bifurcation diagrams of the rf spectra of the dynamics, which have been measured with the ESA. To account for the widely spread characteristic time scales, we present first a zoom into the intermediate frequency range (0-2 GHz, Fig. 4) and second we also give an overview covering the full spectral range of the dynamics until the 22 GHz limit of the ESA (Fig. 5).

The intermediate frequency range $(0-2.5 \mathrm{GHz})$ bifurcation diagram is depicted in Fig. 4. A fine periodic spectral structure first appears over the frequency band analysis, with a small frequency motif of $41 \mathrm{MHz}(1 / T)$. This reveals clearly the signature of the numerous delay modes which are modulating the spectrum. The contrast of this modulation however vanishes progressively as the feedback gain is increased, i.e., when the dynamics is becoming more and more complex. Differently speaking, the nonlinear mixing of the frequency components becomes more and more important, making the $41 \mathrm{MHz}$ periodic modulation smaller and smaller. If we then analyze the global spectral shape evolution over the $\mathrm{GHz}$ scale, we recognize the already described route to chaos: the Hopf bifurcation at $\beta \simeq 0.5$ with an oscillation frequency of ca. FSR/2, the Neimark-Sacker bifurcation at $\beta \simeq 1.3$ with an amplitude modulation governed by the dual time delay $2 T$ and revealed by the side band spectral lines at $\pm(2 T)^{-1}$, the prechaotic transition regimes from $\beta$ $=1.7$ to 1.9 , and the fully developed chaotic regimes starting at 1.9. It is interesting to notice an additional information brought by this spectral analysis and concerning the prechaotic transition regimes. The experimental spectral bifurcation diagram indeed reveals a frequency jump of the main delay oscillating mode around FSR/2 from 1.02 to $1.26 \mathrm{GHz}$ (thus much closer to the exact FSR/2 of the DPSK-d). This jump corresponds approximately to 6 times the delay mode frequency $T^{-1}$. Next, the prechaotic character of the transition regimes is here justified by a significantly increased background frequency noise around the central frequency FSR/2.

The fully developed chaotic regimes starting at $\beta \simeq 2$ can be more finely analyzed with a spectral bifurcation diagram. This is more specifically emphasized with two vertical cuts of the spectral bifurcation diagram, as reported in Fig. 6. At 

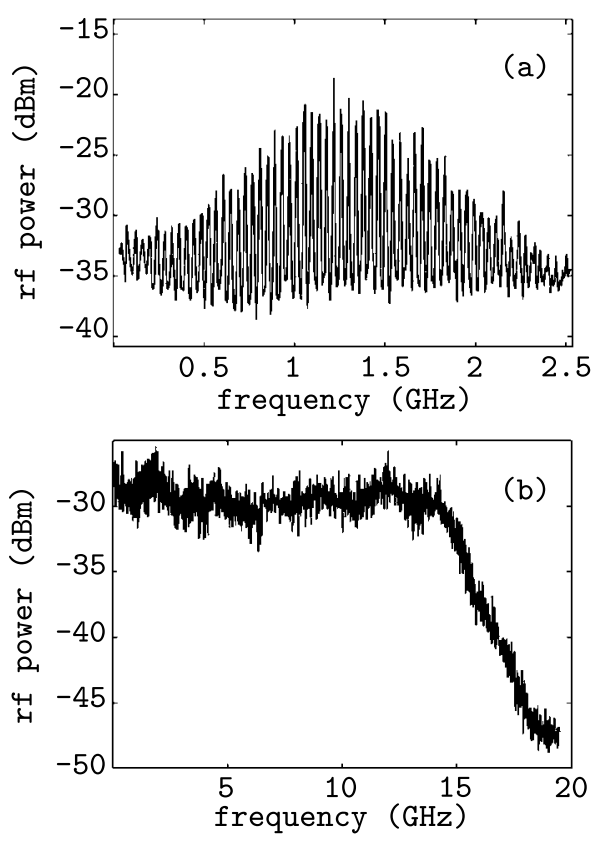

FIG. 6. Vertical cuts of the spectral bifurcation diagram in the fully developed chaotic regimes: (a) $\beta=2.8$ and (b) $\beta=5.1$.

the beginning of this dynamical transition, we first observe a strong spectral contribution of many delay modes together, around half of the FSR frequency (more than 20 delay modes with a power $>-25 \mathrm{dBm}$ ). This high number of delay modes contribution comes together with a globally increased background noise in the frequency intervals between the delay modes [from less than $-45 \mathrm{dBm}$ for $\beta<2$ to more than $>-35 \mathrm{dBm}$ for $\beta>2$; see Figs. 4 and 6(a)]. As $\beta$ is further increased, the fully developed chaotic spectrum becomes flatter and flatter until the maximum achievable experimental gain, where even the rf filtering of the DPSK-d is difficult to identify [see Fig. 6(b)].

Figure 5 shows the high-frequency range bifurcation diagrams of the dynamics spectrum. Both experimental and numerical are represented, to illustrate again the capability of the model to capture the observed dynamics with a relatively good accuracy, when the feedback gain $\beta$ is changed from 0 to 5 . The already-described scenario of the route to chaos is confirmed, highlighting here the role of the DPSK-d FSR in the evolution of the spectrum. As mentioned for the PDF bifurcation diagram, we notice again a difference in the experiment and the numerics for the prechaotic regimes (around $\beta=2$ ): this transition appears as smooth in the numerics, whereas a frequency drift is observed experimentally. This difference is unexplained for the moment. It could be attributed to an already-mentioned particular experimental feature: there is a fine structure of the rf filtering feedback, which can be detected when measuring the open loop linear filtering of the rf feedback. This is not considered in the model, since simulating the same experimentally observed complex ripples (in phase and amplitude) is too difficult to account for in the time domain. The broadband bandpass modeling is indeed smooth and flat in the frequency range of interest, whereas complex small ripples do exist in the actual oscillator filtering feedback. Such ripples are mainly related

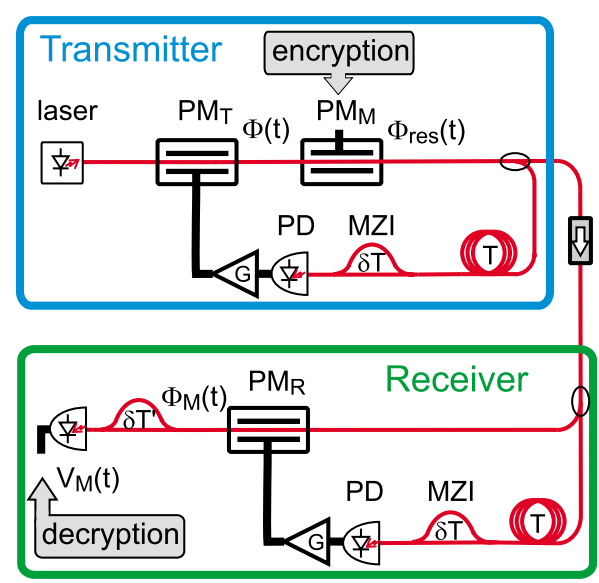

FIG. 7. (Color online) Scheme of a possible phase chaos communication system for encrypted message transmission.

to technological limitations in the realization of flat and ultrabroadband $(30 \mathrm{kHz}-25 \mathrm{GHz})$ electronic amplification developed for high-speed optics communication systems.

Finally, it can be noticed on these figures that for the achievable maximum feedback gain $(\beta=5.1)$, a full bandwidth and nearly flat spectrum is obtained over more than 13 $\mathrm{GHz}$ bandwidth. These spectral features are characteristic of high complexity chaotic dynamics that are highly attractive for applications in high-speed chaos-based communication. Such chaotic optical phase can be used as a noiselike but deterministic carrier signal, which role is to hide a message for encryption purposes and transmission through a fiber channel. An additional important issue in this context is the capability of the proposed architecture to allow for a practical and efficient chaos synchronized receiver, which will be dealt in the last section.

\section{PHASE CHAOS SYNCHRONIZATION FOR CHAOS COMMUNICATION}

Figure 7 depicts a scheme of a possible transmitter and receiver structure, intended for encryption, transmission, and decryption of a chaotic optical phase-encrypted message. The transmitter comprises an additional phase modulator compared to Fig. 1. It is implemented for the mixing of a DPSK-encoded binary message into the broadband chaotic phase modulation demonstrated in the previous section. This phase modulator $\mathrm{PM}_{M}$ can be placed best just behind or before the chaos-generating $\mathrm{PM}_{T}$. For message encryption, the $\mathrm{PM}_{M}$ is modulated by a driving voltage signal (the message) with an amplitude of the order of $V_{\pi}$. The resulting phasemodulated signal $\Phi_{\text {res }}(t)$ at the output of $\mathrm{PM}_{M}$ consists of the message, superimposed to a chaotically modulated optical phase, with a constant intensity. This signal is coupled out of the transmitter using a 50/50 fiber coupler and sent to the receiver. The other part is fed to the MZI followed by a photodiode and subsequently converted into an electrical signal that drives the chaos generating $\mathrm{PM}_{T}$, one delay time $T$ later, as described in Sec. II (nonlinear delayed feedback phase oscillator). In this way, the message is not just simply added onto the chaotic carrier signal, but it is nonlinearly 
mixed with the feedback phase dynamics, according to a chaos masking scheme [30]. This is beneficial regarding security aspects of the communication scheme, since the message itself is blurring the determinism of the stand-alone phase chaos oscillation. Good mixing of the carrier additionally requires that the temporal imbalance of the interferometric element in the feedback loop (MZI) exceeds the characteristic time scale of one message-bit period, $\delta T>T_{\text {bit }}$.

The light output of the transmitter is then sent to the receiver via an optical fiber channel. At the receiver input, the light beam is split into two by a 50/50 fiber coupler; one part is delayed and converted into an intensity signal using an MZI with the same specifications as that of the transmitter. The only difference is that the receiver MZI is tuned (e.g., through the receiver DPSK-d temperature) to operate at an interference phase offset of $\Phi_{R, 0}=\Phi_{T, 0} \pm \pi / 2$ so that the nonlinear $\cos ^{2}$ transformation is performed at the receiver in antiphase, when compared to that of the transmitter. The obtained intensity signal at the output of the receiver MZI is detected, amplified, and used as a driving signal for the receiver phase modulator $\mathrm{PM}_{R}$; the latter PM is practically acting on the second part of the split input light beam. The delay in the receiver needs to be adjusted precisely such that the light that drives the $\mathrm{PM}_{R}$ modulates its own future state at one delay time $T$ later, where $T$ is the delay of the transmitter feedback. In this way, the nonlinear transformation of the phase, previously performed in the transmitter, is reversed and compensated in the receiver, provided that all involved receiver components and operating conditions are matched to that of the transmitter. Fulfilling these requirements, the signal at the output of $\mathrm{PM}_{R}$ only exhibits the message phase modulation that has been previously applied to $\mathrm{PM}_{M}$. The phase-encoded message can then be conventionally converted into an easy-to-detect intensity signal using a second DPSK-d matched with the message bit rate (as implemented in conventional DPSK message transmission schemes).

Following this encoding and decoding architecture adapted for optical phase chaos communication, we successfully demonstrated the synchronization capability of the setup in a back-to-back configuration. The cancellation of the chaotic phase modulation at the receiver (which is a good measure for the synchronization quality) was observed in the optical spectral domain, at the output of $\mathrm{PM}_{R}$, using a highresolution optical spectrum analyzer (OSA) (10 $\mathrm{MHz}$ resolution). Figure 8 reveals in the optical domain both the spectrum of the chaotic signal around the optical carrier wavelength, as well as the residual phase synchronization error measured at the receiver. Both spectra have been recorded at the same point, the output of $\mathrm{PM}_{R}$, so that they exhibit consistent amplitude scaling. The chaotic carrier signal and the synchronization error can be measured by on or off switching the nonlinear delayed optical path in the receiver system, the path which drives the $\mathrm{PM}_{R}$. High synchronization quality is obtained, as it can be noticed, with an error-to-chaos ratio better in average than $10 \mathrm{~dB}$ (in the optical domain, i.e., value theoretically twice less than in the electrical domain) over more than $15 \mathrm{GHz}$. This preliminary result is very encouraging for the next step, the demonstration of high bit rate and high-quality optical communication, involving chaotic phase for masking conventional DPSK sig-

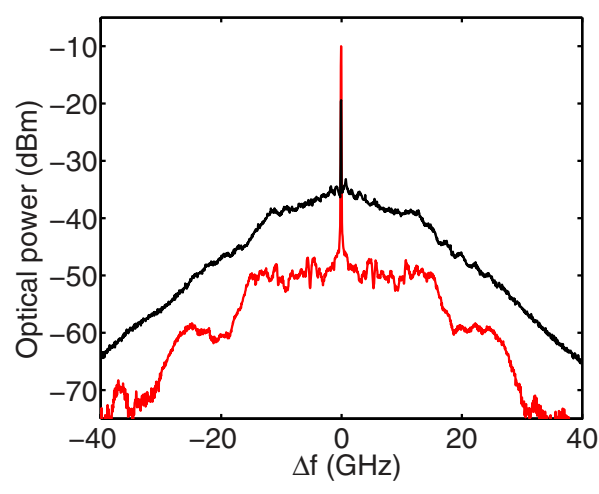

FIG. 8. (Color online) High-resolution optical spectra. Black: chaotic phase modulation spectrum, red: phase chaos cancellation or phase chaos synchronization error.

nal. It is also worth noticing that the proposed synchronized receiver architecture allows for an optical chaos cancellation, which might be a strong advantage of the approach in terms of decoding error level and message recovery quality.

\section{CONCLUSIONS}

An optical phase chaos oscillator was presented. It is constructed following the Ikeda cavity principles, with optoelectronic and electro-optic nonlinear delay feedback architectures. Dynamical features were reported and related to the particularities of the setup, one of which being the multiple delay character: a large delay involved in the feedback and a short delay allowing for a phase-to-intensity nonlinear transformation, with a specific nonlocality in time. When varying the feedback gain, bifurcation diagrams were reported and analyzed in the temporal and spectral domains, revealing an interesting potential of the setup in terms of nonlinear dynamics (variety and complexity of the behaviors) and also in terms of application to optical chaos communications (broadband and fast optical phase chaos). In the latter context, we proposed the experimental use of the optical phase to perform optical chaos communication, which is expected to offer reliable and efficient practical solutions. On the basis of a simple chaos generator architecture, high quality and broadband phase chaos synchronization was demonstrated and a full encoder/decoder scheme was proposed for future investigations of high-speed message transmission using optical phase chaotic carriers. Future work is also oriented toward specific device fabrication for hybrid phase chaos encoder and decoder. More specifically, a laser with two cascaded phase modulators integrated on III-V semiconductor is being designed and explored; it could allow in the future for compact emitter/receiver pairs. Also, the passive nonlinear phaseto-intensity converter could benefit from a customized device approach; this functionality was performed in the present paper with a standard commercial DPSK-d MZI. Customized similar devices with more complex architectures (multiple wave-imbalanced interferometer) would lead to dedicated hardware keys. Such a hardware key could offer efficient flexibility in the definition of the physical parameters that are required for chaos generation, for sensitive and accurate 
chaos synchronization, and for the development of efficient and secure optical chaos communication systems.

\section{ACKNOWLEDGMENTS}

This work was mainly conducted in the frame of the European PICASSO Project (Project No. FP6-2006-IST-
2.5.1) and partly within the French National project ACSCOM. The authors also thank the Region FrancheComte for its support of advanced optical telecommunication research program within the FEMTO-ST institute. L.L. and J.D. are grateful to the Institut Universitaire de France for its important institutional support.
[1] V. Annovazzi-Lodi, S. Donati, and A. Sciré, IEEE J. Quantum Electron. 33, 1449 (1997).

[2] P. Colet and R. Roy, Opt. Lett. 19, 2056 (1994).

[3] C. R. Mirasso, P. Colet, and P. Garcia-Fernandez, IEEE Photon. Technol. Lett. 8, 299 (1996).

[4] R. Lang and K. Kobayashi, IEEE J. Quantum Electron. 16, 347 (1980).

[5] T. Mukai and K. Otsuka, Phys. Rev. Lett. 55, 1711 (1985).

[6] Y. Cho and T. Umeda, IEEE J. Quantum Electron. 59, 131 (1986).

[7] Fundamental Issues of Nonlinear Laser Dynamics, edited by B. Krauskopf and D. Lenstra, AIP Conf. Proc. No. 548 (AIP, New York, 2000).

[8] G. D. VanWiggeren and R. Roy, Science 279, 1198 (1998).

[9] J.-P. Goedgebuer, L. Larger, and H. Porte, Phys. Rev. Lett. 80, 2249 (1998).

[10] I. Fischer, Y. Liu, and P. Davis, Phys. Rev. A 62, 011801(R) (2000).

[11] H. Abarbanel, M. Kennel, L. Illing, S. Tang, H. Chen, and J. Liu, IEEE J. Quantum Electron. 37, 1301 (2001).

[12] A. Argyris, M. Hamacher, K. E. Chlouverakis, A. Bogris, and D. Syvridis, Phys. Rev. Lett. 100, 194101 (2008).

[13] H. M. Gibbs, F. A. Hopf, D. L. Kaplan, and R. L. Shoemaker, Phys. Rev. Lett. 46, 474 (1981).

[14] A. Neyer and E. Voges, IEEE J. Quantum Electron. 18, 2009 (1982).

[15] L. Larger, J.-P. Goedgebuer, and J.-M. Merolla, IEEE J. Quantum Electron. 34, 594 (1998).

[16] K. Ikeda, Opt. Commun. 30, 257 (1979).

[17] N. Gastaud, S. Poinsot, L. Larger, M. Hanna, J.-M. Merolla, J.-P. Goedgebuer, and F. Malassenet, Electron. Lett. 40, 898 (2004).
[18] A. Argyris, D. Syvridis, L. Larger, V. Annovazzi-Lodi, P. Colet, I. Fischer, J. Garcia-Ojalvo, C. R. Mirasso, L. Pesquera, and A. K. Shore, Nature (London) 438, 343 (2005).

[19] The term phase synchronization is concerned in this paper by a self-referenced laser beam, which is electro-optically phase modulated via a chaotic delayed feedback loop. Standard telecom differential optical phase modulation is thus involved. In [20], optical phase synchronization is explored in the sense of the relative phase coherence of two distinct lasers, which is a different situation than the one concerned here.

[20] Y. Aviad, I. Reidler, W. Kinzel, I. Kanter, and M. Rosenbluh, Phys. Rev. E 78, 025204(R) (2008).

[21] M. Le Berre, É. Ressayre, A. Tallet, and H. M. Gibbs, Phys. Rev. Lett. 56, 274 (1986).

[22] B. Dorizzi, B. Grammaticos, M. Le Berre, Y. Pomeau, É. Ressayre, and A. Tallet, Phys. Rev. A 35, 328 (1987).

[23] R. Vicente, J. Daudén, P. Colet, and R. Toral, IEEE J. Quantum Electron. 41, 541 (2005).

[24] M. Peil, M. Jacquot, Y. K. Chembo, L. Larger, and T. Erneux, Phys. Rev. E 79, 026208 (2009).

[25] Y. Chembo, L. Larger, and P. Colet, IEEE J. Quantum Electron. 44, 858 (2008).

[26] D. Pieroux, T. Erneux, T. Luzyanina, and K. Engelborghs, Phys. Rev. E 63, 036211 (2001).

[27] J. N. Blakely, L. Illing, and D. J. Gauthier, IEEE J. Quantum Electron. 40, 299 (2004).

[28] K. Ikeda, K. Kondo, and O. Akimoto, Phys. Rev. Lett. 49, 1467 (1982).

[29] Y. C. Kouomou, P. Colet, L. Larger, and N. Gastaud, IEEE J. Quantum Electron. 41, 156 (2005).

[30] C. R. Mirasso, J. Mulet, and C. Masoller, IEEE Photon. Technol. Lett. 14, 456 (2002). 